\title{
Efficacy of abdominal dermis fat graft as the interpositional material in the management of Temporomandibular joint ankylosis in children- an original study
}

\author{
${ }^{1}$ Dr. S.Gandhiraj MDS, ${ }^{2}$ Dr.Shubra chauhan MDS, ${ }^{3}$ Dr.Prakash MDS
}

\begin{abstract}
Aim of the study is to evaluate the efficacy of inter positional dermis-fat graft in the management of Temporo-mandibular joint(TMJ) ankylosis in children by assessing the maximal mouth opening prior and after procedure, complication at both donor and recipient sites, and prevention of re-ankylosis after inter positioning. Both unilateral, bilateral osseous and fibro-osseous ankylosis of both sexes in 5 patients and the age ranging from 5-12 years were included in this study. All the cases were treated with gap arthroplasty and the gap was filled with an autogenous abdominal dermis-fat graft procured from the supra pubic region. The dermis fat graft interpositional arthroplasty is a safe and effective procedure in the management of temporo-mandibular joint ankylosis which prevents re-ankylosis especially in children by minimizing the occurrence of excessive joint fibrosis and heterotopic calcification. .
\end{abstract}

Key words: Abdominal dermis fat graft, Interpositional gap arthroplasty, TMJ gap arthroplasty

\section{Introduction}

TMJ ankylosis is an extremely disabling affliction that causes problem in mastication, digestion, speech, appearance and hygiene. It also has an impact on the psychologic development of patient and can place patient's life in jeopardy at anytime because of the inability to open the mouth. More number of authors worked on the placement of temporalis muscle as the interposition material in the TMJ (temporomandibular joint) surgery. Verneuil [1] was the first to use autogenous tissue (temporalis flap) as an interpositional material in 1860. In 1914, Murphy [2] reported the use of autogenous fat for interposition after lysis of TMJ ankylosis. Inherent lack of strength and profound atrophic tendencies account for lack of support for this tissue at that time. The use of temporalis muscles as the interposition material in the TMJ surgery have their own merits and demerits. Arthroplasty without interposition requires a gap of 10-20 millimetres to prevent re-ankylosis. With this large gap, there is loss of ramus height, no support for the rotating mandible with a tendency to contra lateral open bite and deviation on opening. So creation of large gap is not an ideal method of management for TMJ ankylosis, a minimal gap not more than $10 \mathrm{~mm}$ with interposition material become mandatory to prevent re ankylosis. Most of the operators accepted the temporalis muscle and facia are the right choice as the interposition material for the past few decades. Difficulty in mouth opening and vigorous post operative exercise are troublesome for the patient if temporalis muscle is used as the interposition material due to compression of the nerves which may subsequently reduce the mouth opening. Balaji SM in 2003 [3] found the submandibular anchorage of the broad temporalis muscle in the prevention of re ankylosis. He also said the temporalis flap is the ideal interposition material due to its close proximity, minimal risk of nerve damage and with increased vascularity. He stressed that postoperative exercise is mandatory but to a minimal extent only.Though many advantages were claimed by using these flaps, discomforts like pain during movement due to nerve compression and an ugly bulging in the temporal region of that side may not be neglected. Steinberg and Hohn in the year 1994[4] reported two cosmetically advantageous sites (suprapubic and inguinal fold) for harvesting dermal graft with primary linear closure. Chossegros et al in 1999 [5] in a retrospective review with 31 patients concluded the reliability of full thickness skin graft interposition. He observed $90 \%$ success and also recommended the necessity for early physiotheraphy, and about the right selection of interposition material for preventing recurrence. G. Dimitroulis in his retrospective clinical study in 2004 [6] found that autogenous dermis-fat interpositional graft is an effective procedure for the prevention of re-ankylosis. Dimitroulis $G$ et al again in 2008[7], after detailed studies reported about the fate of dermal fat graft by using magnetic resonance image. They have stated that the intermittent compressive forces of the joint may act as a negative influence on the growth and also explained the fat tissues are maintained within the joint space itself. Larry M Wolford et al in 2008, [8] in their study of fixing prosthesis in the joint space have not encountered any fibrosis or bone formation. Mehothra et al in 2008 [9] randomly divided 17 patients in to 2 groups, the first group was treated with dermis fat graft procured from the groin where as the controlled group treated with temporalis fascia. They concluded the use of dermal fat graft has minimal donor site morbidity which is a safe and effective inter position material to prevent reankylosis. Yazdani et al in 2010[11] conducted a study on 20 patients to prove the efficacy of Temporalis muscle 
interposition verses dermal fat grafts which were implemented on 2 groups, 10 patients in each group, by considering the amount of presurgical and post surgical maximum incisal mouth opening and lateral excursion. The result was comparable and almost satisfactory. Thangavel et al in 2011 [12] in their study on seven patients with dermal fat graft interposition, reported that they have not witnessed any relapse during the 2 years follow up. G.Dimitroulis et al in 2011[14] reported that Neo adipogenesis inhibit the growth of new bone and cartilage there by inhibit the regeneration of the new condylar head, but the fat in that site failed to protect the condyle completely against additional deteriration in $6.5 \%$ of the total 17 patients. Virendra singh et al in 2011 [15] in a retrospective study of 10 ankylosis patients with a follow up of 6 months to 2 years, have not witnessed any relapse during the treatment using buccal pad fat as an interposition material.. Gaba et al in 2012 [16]in a study with 18 patients achieved better mouth opening, less pain and prevention of the heterotropic bone formation as the advantages of using buccal pad fat . The complications like scar, infection, seroma and wound dehiscence are similar to those encountered with other graft procurement technique and may be minimized by adherence to proper surgical technique. In this study, no post operative complications were recorded. All our patients showed satisfactory healing at the donor site. No patient encountered any decrease in mouth opening till the end of 2 years follow up.

\section{Materials and methods :}

A prospective study involving 5 children who presented with TMJ ankylosis of both sexes and the age ranging from 5-12 years were included in this study. Both unilateral, bilateral osseous or Fibroosseous ankylosis, were included in this study. Selection criteria are patients with restricted (or) nil mouth opening, radiological evidence of TMJ ankylosis, history of previous surgery presenting with recurrence. Patient with evidence of fibrous ankylosis, false ankylosis due to extra articular fibrosis, pathological condition or mechanical obstruction were not included in this study.

Alkayat-Bramley incision was used to gain access to the TMJ in all our cases. The ankylosed segment was removed using upper and lower osteotomy cuts and a gap about $10 \mathrm{~mm}$ was created (Fig. 1). Adequate mouth opening ranging from 31 to $36 \mathrm{~mm}$ was achieved. No attempt was made to refashion the glenoid fossa. The dermal fat graft was procured from the suprapubic region. An elliptical incision of about $7 \times 3 \mathrm{~cm}$ was made in the skin to harvest the graft in the suprapubic region.(fig 2) This area was deepithelised. A second elliptical incision was made just within the borders of the original incision to procure dermis-fat graft of volume $6-20 \mathrm{ml}$ (Fig. 3). This volume is about $20-30 \%$ more than the actually required for the interposition. The graft was stored in the saline solution. The wound margins were undermined and layer-wise closure was performed. The graft was folded onto itself with the dermis surfaces apposed. The subcutaneous fat was neatly trimmed to fill the gap making sure that all the dead spaces were eliminated. The graft was passively placed into the cavity (Fig. 4). The capsule was then closed and wound repaired in layers. Physiotherapy, help to mobilize the joint, was commenced 7 days after operation and continued for 4-6 week. The clinical evaluation of efficacy of this procedure was done on the basis of maximum mouth opening studied as pre procedural, intra operative, one-week post procedural, and monthly follow-up period ranging from 21 to 24 months. The other parameters considered were prevention of reankylosis after interpostioning and complications at both donor and recipient site. Patients who underwent gap arthroplasty with dermis fat graft interposition had an significant increase in mouth opening from a range of 2 to $7 \mathrm{~mm}$ (mean 4.4) prior to procedure to $31-36 \mathrm{~mm}$ (mean $34 \mathrm{~mm}$ ) following surgical intervention (table 1 , Fig. 5,6). This mouth opening achieved on the table was maintained till the end of 2 years follow up. All the unilateral ankylosis patients had some degree of mandibular deviation to the operated side on maximum mouth opening. Only one unilateral case (case no.3) necessitated ipsilateral coronoidectomy, because the mouth opening achieved with the gap arthroplasty was judged to be insufficient at the time of surgery and so coronoidectomy performed during the same operation. In the follow up period, none of the patients suffered from reankylosis. It is of interest that all patients were able to make protrusive and lateral movements within a couple of month following surgery. All the patients had a satisfactory mouth opening and were pleased with the outcome. Post operatively no reduction in mouth opening was witnessed in any of the patient. None of the patients requested for scar revision over the supra pubic region.

\section{Discussion}

In India, trauma to the TMJ was documented as a major etiologic factor for TMJ ankylosis. [10] In this study, all the patients had a history of trauma. Lot of autogenous, allogenic, alloplastic and xenografts materials are used as the interpositional materials since from the earlier days. The autogenous materials commonly used are muscle, fascia, cartilages, skin, fat etc for the management of TMJ ankylosis. A live pedicled graft is more successfully helping in the prevention of recurrence. Temporalis muscle and fascia are broadly used by many surgeons as the interposition material. Post surgical pain on jaw movements and a vigorous physiotheraphy to prevent reankylosis are the inevitable problems in patients who are treated with 
temporalis muscle as interposition material.[3,10,13] Balaji SM explained in his modified technique that less amount of post operative pain and decreased need for post operative physiotheraphy are the advantages. [3] To overcome the disadvantages pertaining to temporalis muscle use in gap arthroplasty a safe and effective procedure ,interposition with dermal fat graft [4,6] is implimented . Quick and easy graft procurement, minimal donor site morbidity, easy sculpture to fit neatly into any size cavity, hidden scar (below the belt line) and minimizes the occurrence of excessive joint fibrosis and heterotopic calcification there by consequently providing improved range of motion are said to be the advantages of using dermal fat graft. [3, 4, 69,12$]$ Pain on jaw movements are almost alleviated as the physiotherapy started 1week after operation. This sort of advantage helps the children to continue the exercise without a break which in turn helps in prevention of re ankylosis. Viable condition of fat tissues even after years at the joint region are studied and proved by many authors. $[6,7,14,16,17]$ The surgeons who used the dermal fat graft as the interposition material for the management of TMJ ankylosis uniformly claimed the minimal fibrosis action and hetarotopic calcification prevention $[8,14]$ as the advantages of using dermis fat graft. Some authors also practiced with the buccal pad fat $[15,16]$ as the interposition material and uniformly claimed the material, buccal pad fat, was taking appreciable part in the prevention of reankylosis. Satisfactory results were obtained by surgeons who used dermal fat grafts rather than using the usual temporalis fascia.[11] The interpositioned fat tissues nicely thrives and adopted to the cavity and allows the functional movement and the viable fat tissues help to prevent reankylosis as the fat tissues in the cavity are living even for many years [14,17]. The purpose of this article is to present the clinical experience of using autogenous abdominal dermis-fat interpositional graft in the surgical management of TMJ ankylosis especially in children [9] where as majority of the other studies with interposition dermal fat grafts were conducted on adult patients. In this study, we preferred to wait, for a period of 7 days before commencing mobilization of mandible. This delay allows early phase healing of the surrounding tissue and interpositional graft, which may perhaps respond more favourably to mobilization through pseudo-articular joint formation than with immediate post operative mobilization. Post operative physiotheraphy for a long period is a must in order to maintain the mouth opening achieved during surgery and for prevention of recurrence. Unfortunately lack of physiotheraphy is seen in child patients, whose joints are inter positioned with temporalis muscle, due to pain, as the sensory innervations are compressed during jaw motion and physiotharaphy. This is a cause for gradual decrease in the mouth opening which may lead to relapse. But pain at the surgery site during jaw movements and physiotheraphy is completely absent in our patients. Postoperatively there was no need necessitated for our patients to keep the jaw idle without doing mouth opening exercise, as there was no pain associated with the TMJ region during movement. Normal regular movement of the jaw promote dramatic improvement in mouth opening and no reduction in mouth opening was encountered during the 2 years follow up. Therefore the dermis fat graft interposition is an effective and ideal procedure for the treatment of TMJ ankylosis in children.

\section{Figure and table}

\section{V.Figures :}

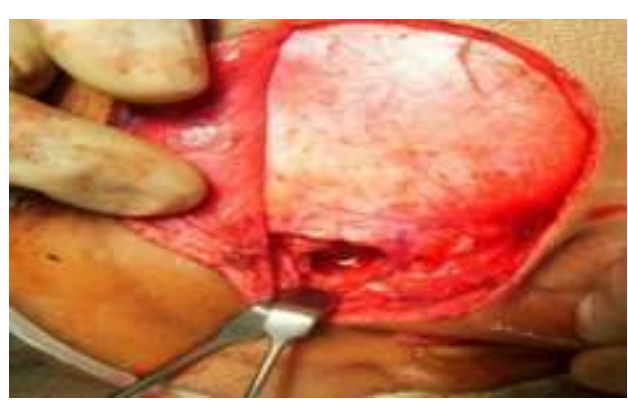

Fig.1:Gap created after ankylotic mass resection

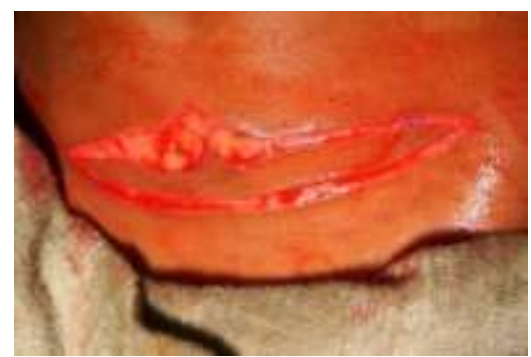

Fig.2:Incision made in the suprapubic region 


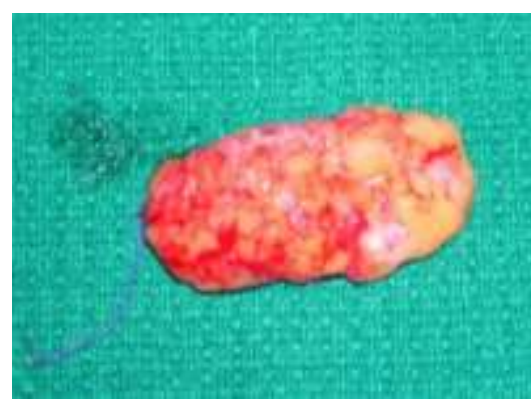

Fig.3:Harvested dermis-fat graft

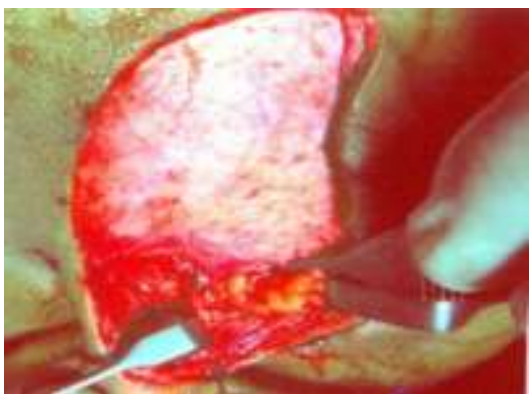

Fig.4: Interposition of dermis-fat graft

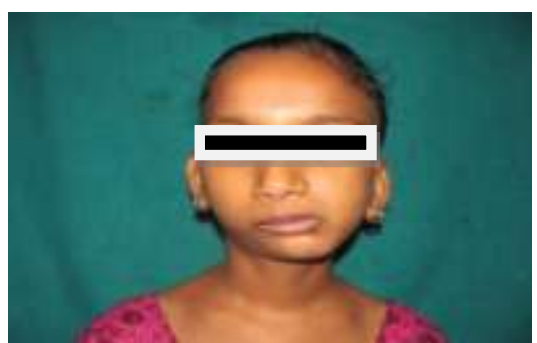

Fig.5: Preoperative mouth opening

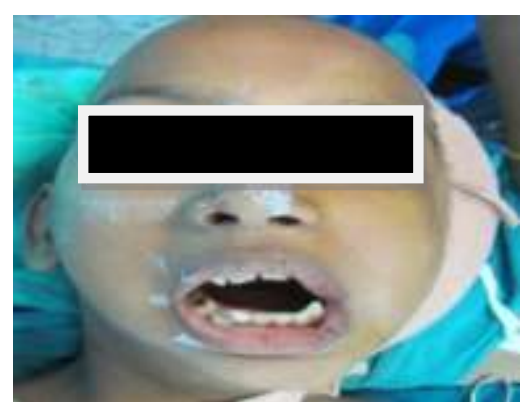

Fig.6:Post operative mouth opening

IV. Table 1 preoperative, on table and post operative mouth opening after 2 years

\begin{tabular}{|c|c|c|c|c|c|c|c|c|}
\hline \multirow{2}{*}{$\begin{array}{c}\text { Patient } \\
\text { No }\end{array}$} & \multirow[t]{2}{*}{ Age } & \multirow[t]{2}{*}{ Sex } & \multirow{2}{*}{$\begin{array}{c}\text { Joint } \\
\text { affected }\end{array}$} & \multirow{2}{*}{$\begin{array}{l}\text { Duration of } \\
\text { Ankylosis } \\
\text { (Years) }\end{array}$} & \multirow{2}{*}{$\begin{array}{c}\text { Follow up } \\
\text { period }\end{array}$} & \multicolumn{3}{|c|}{ Mouth opening in $\mathrm{mm}$} \\
\hline & & & & & & $\begin{array}{l}\text { Pre .op } \\
\text { opening }\end{array}$ & $\begin{array}{l}\text { Post op } \\
\text { opening }\end{array}$ & $\begin{array}{l}\text { Mouth opening } \\
\text { At } 2 \text { years }\end{array}$ \\
\hline 1 & 12 & $\mathrm{M}$ & $\mathrm{L}$ & 4 & $\begin{array}{c}24 \\
\text { months }\end{array}$ & $7 \mathrm{~mm}$ & $36 \mathrm{~mm}$ & $36 \mathrm{~mm}$ \\
\hline 2 & 5 & $\mathrm{M}$ & Both & 1 & $\begin{array}{c}22 \\
\text { months }\end{array}$ & $3 \mathrm{~mm}$ & $35 \mathrm{~mm}$ & $35 \mathrm{~mm}$ \\
\hline 3 & 10 & $\mathrm{~F}$ & $\mathrm{R}$ & 2 & $\begin{array}{c}23 \\
\text { months }\end{array}$ & $6 \mathrm{~mm}$ & $31 \mathrm{~mm}$ & $31 \mathrm{~mm}$ \\
\hline 4 & 12 & $\mathrm{M}$ & $\mathrm{R}$ & 2 & $\begin{array}{c}21 \\
\text { months }\end{array}$ & $2 \mathrm{~mm}$ & $35 \mathrm{~mm}$ & $35 \mathrm{~mm}$ \\
\hline 5 & 9 & $\mathrm{~F}$ & $\mathrm{R}$ & 3 & $\begin{array}{c}24 \\
\text { months }\end{array}$ & $4 \mathrm{~mm}$ & $33 \mathrm{~mm}$ & $33 \mathrm{~mm}$ \\
\hline
\end{tabular}




\section{Conclusion:}

The dermis fat graft interpositional gap arthroplasty is a safe and effective procedure in the management of TMJ ankylosis especially in children. The tendancy for reankylosis are more common among children, due to lack of exercise due to because of fear for pain during jaw motion and physiotheraphy, if temporalis muscle is used as the interposition material. The jaw movements and physiotheraphy are well tolerated and maintained by our patients since the gaps were filled with dermis fat grafts. The dermis fat graft minimizes the occurrence of excessive joint fibrosis and heterotopic calcification and consequently providing improved range of motion. Graft procurement is quick and easy with minimal morbidity and the wound and scar in the anatomical skin creases are accepted cosmetically. The results obtained in our study are highly satisfactory and encouraging, supporting the role of dermis fat graft as the interposition material in TMJ ankylosis cases especially in children. However, a large sample size and a longer follow-up period are required to consolidate the findings.

\section{References}

[1] Verneuil, A.: Archives de Medecine, 1860, p. 284

[2] John B. Murphy. The Classic: Ankylosis: Arthroplasty-Clinical and Experimental Clin Orthop Relat Res. 2008 November; 466(11): 2573-2578. Published online 2008 August 30. doi: 10.1007/s11999-008-0455-5.PMCID: PMC2565041

[3] Balaji SM. .Modified temporalis anchorage in craniomandibular reankylosis Int J Oral Maxillofac Surg. 2003 Oct;32(5):480-5

[4] Steinberg MJ, Hohn FI. Procurement of dermal graft from the suprapubic or inguinal fold region with primary linear closure J Oral Maxillofac Surg. 1994 Aug;52(8):813-6

[5] Chossegros C, Guyot L, Cheynet F, Blanc JL, Cannoni P. Full-thickness skin graft interposition after temporomandibular joint ankylosis surgery: a study of 31 cases. Int J Oral Maxillofac Surg. 1999;28:330-334

[6] G. Dimitroulis. The interpositional dermis-fat graft in the management of temporomandibular joint ankylosis. International Journal of Oral \& Maxillofacial Surgery Volume 33, Issue 8, Pages 755-760, December 2004

[7] Dimitroulis G, Trost N, Morrison W. The radiological fate of dermis-fat grafts in the human temporomandibular joint using magnetic resonance imaging. Int J Oral Maxillofac Surg 2008 Mar;37(3):249- 54. doi 10.1016/j.ijom.2007.11.017. Epub 2008 Feb 13 PMID:18276114

[8] Larry M. Wolford, Carlos A. Morales-Ryan, Patricia Garcia Morales, and Daniel Serra Cassano. Autologous fat grafts placed around temporomandibular joint total joint prostheses to prevent heterotopic bone formation Proc (Bayl Univ Med Cent). 2008 July; 21(3): 248-254. Copyright @ 9 2008, Baylor University Medical Center PMCID: PMC2446413

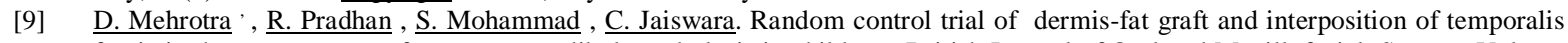
fascia in the management of temporomandibular ankylosis in children. British Journal of Oral and Maxillofacial Surgery Volume 46, Issue 7, October 2008, Pages 521-526

[10] Mohammad Bayat, Amirali Badri,,Nima Moharamnejad. Treatment of temporo mandibular joint ankylosis: gap and interpositional arthroplasty with temporalis muscle flap. Oral and Maxillofacial Surgery. December 2009, Volume 13, Issue 4, pp 207- 212

[11] Yazdani, Javad; Ali Ghavimi, Mohammad ; Pourshahidi, Sara ; Ebrahimi, Hooman. Comparison of Clinical Efficacy of Temporalis Myofascial Flap and Dermal Graft as Interpositional Material in Treatment of Temporomandibular Joint Ankylosis. Journal of Craniofacial Surgery: July 2010 - Volume 21 - Issue 4 - pp 1218-1220 doi:10.1097/SCS.0b013e3181e1b4f6

[12] Thangavel K. Santhosh KumarA. Vaidhyanathan M. Balaji R. Narendar. Versatility of full thickness skin-subcutaneous fat grafts as interpositional material in the management of temporomandibular joint ankylosis International Journal of Oral \& Maxillofacial Surgery Volume 40, Issue 1, Pages 50-56, January 2011

[13] Yadavalli Guruprasad, Dinesh Singh Chauhan, and K. M. Cariappa. Retrospective Study of Temporalis Muscle and Fascia Flap in Treatment of TMJ AnkylosisJ Maxillofac Oral Surg. 2010 December; 9(4): 363-368. Published online 201 February 4. doi: 10.1007/s12663-010-0139-z PMCID: PMC3177478A

[14] G. Dimitroulis J. Slavin,W. Morrison. Histological fate of abdominal dermis-fat grafts implanted in the temporomandibular joint of the rabbit following condylectomy International Journal of Oral \& Maxillofacial Surgery Volume 4, issue 2, Pages 177183, February 2011

[15] Virendra Singh, Rahul Dhingra, Bindu Sharma, Amrish Bhagol, Prashant Kumar. Retrospective Analysis of Use of Buccal Fat Pad as an Interpositional Graft in Temporomandibular Joint Ankylosis: Preliminary Study. Journal of Oral and Maxillofacial Surgery Volume 69, Issue 10, Pages 2530-2536, October 2011

[16] Gaba S, Sharma RK, Rattan V, Khandelwal N. The long-term fate of pedicled buccal pad fat used for interpositional arthroplasty in TMJ ankylosis J Plast Reconstr Aesthet Surg 2012 Nov;65(11):1468-73. doi: 10.1016/j.bjps.2012.05.016. Epub 2012 Jun 12.

[17] George Dimitroulis. Macroscopic and Histologic Analysis of Abdominal Dermis-Fat Grafts Retrieved From Human Temporomandibular Joints. Journal of Oral and Maxillofacial Surgery Volume 69, Issue 9 , Pages 2329-2333, September 2011 